\title{
Correlation between parameters at initiation of renal replacement therapy and outcome in patients with acute kidney injury
}

\author{
Marlies Ostermann ${ }^{1}$ and René WS Chang ${ }^{2}$
}

\author{
1Department of Critical Care, Guy's \& St Thomas' Foundation Hospital, Westminster Bridge Road, SE1 7EH, UK \\ 2Department of Nephrology \& Transplantation, St George's Hospital, Blackshaw Road, SW17 0OT, UK \\ Corresponding author: Marlies Ostermann, Marlies@ostermann.freeserve.co.uk
}

Received: 17 Aug 2009 Revisions requested: 7 Oct 2009 Revisions received: 26 Oct 2009 Accepted: 4 Nov 2009 Published: 4 Nov 2009

Critical Care 2009, 13:R175 (doi:10.1186/cc8154)

This article is online at: http://ccforum.com/content/13/6/R175

(C) 2009 Ostermann and Chang et al.; licensee BioMed Central Ltd.

This is an open access article distributed under the terms of the Creative Commons Attribution License (http://creativecommons.org/licenses/by/2.0), which permits unrestricted use, distribution, and reproduction in any medium, provided the original work is properly cited.

\begin{abstract}
Introduction Renal replacement therapy (RRT) is a fully established treatment for critically ill patients with acute kidney injury (AKI) but there are no scientifically established criteria when to initiate it. Our objectives were to describe the epidemiology of critically ill patients with AKI receiving RRT and to evaluate the relationship between biochemical, physiological and comorbid factors at time of RRT and ICU mortality.
\end{abstract}

Methods Retrospective analysis of demographic and physiologic data of 1,847 patients who received RRT for AKI in 22 ICUs in UK and Germany between 1989 - 1999.

Results $54.1 \%$ of RRT patients died in ICU. ICU survivors were younger, had a lower APACHE II score and fewer failed organ systems on admission to ICU compared to non-survivors. Multivariate analysis showed that at time of initiation of RRT, independent risk factors for ICU mortality were mechanical ventilation [odds ratio (OR) 6.03], neurological failure (OR 2.48), liver failure (OR 2.44), gastrointestinal failure (OR 2.04), pre-existing chronic illnesses (OR 1.74), haematological failure (OR 1.74), respiratory failure (OR 1.62), oligoanuria (OR 1.6), age (OR 1.03), serum urea (OR 1.004) and cardiovascular failure (OR 1.3). A higher $\mathrm{pH}$ at initiation of RRT was independently associated with a better outcome. Failure to correct acidosis and development of more organ failure within 48 hours after initiation of RRT were also associated with an increased risk of dying in ICU.

Conclusions Oligoanuria, acidosis and concomitant dysfunction of other organs at time of RRT were associated with poor survival. In contrast, serum creatinine and urea levels only had a weak correlation with outcome after RRT.

\section{Introduction}

Acute kidney injury (AKI) is a common problem in hospitalised patients with a reported incidence of between 10 and $20 \%$ but as high as $70 \%$ in critically ill patients in the intensive care unit (ICU) [1-4]. To date, there are no curative therapies. Management is limited to fluid and haemodynamic optimisation, and renal replacement therapy (RRT) when necessary. Furthermore, there are no robust data to accurately distinguish in advance between injured kidneys that will need extracorporeal support and kidneys that retain capacity for early recovery.

Although RRT has been an integral part of critical care for decades and technologies have advanced considerably, there are no scientifically established criteria for the initiation of RRT. As a result, the provision of renal support is very variable in clinical practice [5-7].

The indications for RRT in critically ill patients with AKI have generally been extrapolated from the end-stage kidney disease experience and included refractory hyperkalaemia, resistant fluid overload, severe persistent metabolic acidosis, and overt uraemic symptoms, including uraemic pericarditis and encephalopathy. Although there is little dispute about the necessity of RRT for these urgent indications, there is no consensus on the degree of azotaemia or the duration of AKI that warrants RRT in the absence of these 'absolute' indications [8]. Clinical studies addressing the 'optimal' timing of RRT are conflicting [9-12]. In a meta-analysis, Seabra and colleagues

AKI: acute kidney injury; APACHE: acute physiology and chronic health evaluation score; Cl: confidence interval; HD: intermittent haemodialysis; ICU: intensive care unit; OR: odds ratio; pCO2: partial pressure of carbon dioxide; PD: peritoneal dialysis; RRT: renal replacement therapy; SOFA: Sequential Organ Failure Assessment. 
summarised the results of 23 studies, including four randomised controlled trials, which compared the effect of "early" versus "late" RRT on mortality in patients with AKI [12]. Despite the conclusion that early institution of RRT might have a beneficial effect on survival, the authors emphasized that the studies were very heterogenous and differed in quality. The differentiation between 'early' and 'late' RRT is variable and usually based on arbitrary thresholds in traditional parameters such as serum creatinine or urine output, time from admission to ICU or time from diagnosis of AKI [11].

In 2006, the AKI Network assembled a multidisciplinary stakeholder committee with representation from the 18 leading international professional societies of critical care and nephrology. They identified the key questions for future research in the field of AKI in ICU [13]. Top priority was given to the broad topics of epidemiology of AKI and RRT, including the quest for criteria for RRT.

The objectives of this study were twofold. Firstly, to describe the epidemiology of ICU patients treated with RRT and to compare them with AKI stage III patients who did not receive RRT. Secondly, in search of the optimal criteria for RRT, we aimed to evaluate the relation between different physiological, metabolic and comorbid factors at the time of initiation of RRT and subsequent outcome.

\section{Materials and methods Study population}

Using the Riyadh Intensive Care Program database with demographic and daily physiological data of 41,972 adult patients admitted to $19 \mathrm{ICUs}$ in the UK and three ICUs in Germany between June 1989 and October 1999, we analysed the data of 1847 patients who had received RRT for AKI.

Receiving RRT is one of four criteria for the diagnosis of AKI stage III according to the AKI network [14]. For comparison, we identified 935 patients with AKI stage III as defined by the creatinine criteria (ie. rise in serum creatinine to $\geq 354 \mu \mathrm{mol} / \mathrm{L}$ or rise in serum creatinine by $>300 \%$ from baseline within 48 hours) who were not treated with RRT.

All 22 centres included in the study were able to provide RRT if necessary.

\section{Data analysis}

Acute severity of illness was measured using the acute physiology and chronic health evaluation score (APACHE) II and Sequential Organ Failure Assessment (SOFA) scoring system. Organ system failures were determined according to the criteria by Knaus and colleagues [15].

Cardiovascular failure was defined by the presence of one or more of the following: heart rate 54 beats/min or less; mean arterial blood pressure less than $50 \mathrm{mmHg}$; occurrence of ven- tricular tachycardia and/or ventricular fibrillation; serum $\mathrm{pH}$ of 7.24 or less with a partial pressure of carbon dioxide $\left(\mathrm{pCO}_{2}\right)$ of $49 \mathrm{mmHg}$ or less.

Respiratory failure was defined as a presence of one or more of the following: respiratory rate of 5 breaths $/ \mathrm{min}$ or less or 49 breaths/min or more; $\mathrm{pCO}_{2} 50 \mathrm{mmHg}$ or more; alveolar-arterial $\mathrm{pO}_{2}$ difference of $350 \mathrm{mmHg}$ or more; dependent on ventilator on the fourth day of organ system failure (i.e. not applicable for the initial 72 hours of organ system failure).

Haematological failure was defined as the presence of one or more of the following: white blood cells of 1000 cells $/ \mathrm{mm}^{3}$ or less; platelets of 20,000 cells $/ \mathrm{mm}^{3}$ or less; haematocrit of $20 \%$ or less.

Neurological failure was defined as Glasgow Coma Score of 6 or less (in absence of sedation). If patient is sedated and/or paralysed, neurological scoring is not performed and patient is not considered in neurological failure.

Hepatic failure was defined by the presence of both: bilirubin more than $60 \mathrm{mg} / \mathrm{L}$ or a two-fold increase in serum alkaline phosphatase; prothrombin time more than four seconds over upper limit of normal range or a two-fold increase in serum aspartate aminotransferase.

We also included a definition for gastrointestinal failure, which was 'failure to tolerate enteral nutrition and need for total parenteral nutrition' [16].

The highest number of failed organs (excluding renal failure) on any day during the stay in the ICU was recorded as 'Maximum number of associated organ failure'.

We also recorded whether patients had evidence of any of the following pre-existing chronic illnesses:

Cardiovascular - New York Heart Association Class IV angina or symptoms at rest or on minimal exertion, e.g getting dressed or self-care;

Liver - Biopsy proven cirrhosis and documented portal hypertension or previous episodes of variceal bleed/liver failure/ encephalopathy;

Respiratory - Chronic restrictive, obstructive, or vascular disease resulting in severe exercise restrictions, e.g. unable to climb stairs or documented chronic hypoxia, hypercapnea, secondary polycythaemia, severe pulmonary hypertension $(>40 \mathrm{mmHg}$ ) or respiratory dependency;

Immunocompromised - Patients receiving immunosuppression, chemotherapy, radiation, long-term steroid treatment, leukaemia, lymphoma, AIDS or widespread metastatic cancer. 


\section{Ethics approval}

The local ethics committee confirmed that Research and Ethics approval was not required for this study. The need for informed consent was also waived because the study required neither an intervention nor breach of privacy or anonymity.

\section{Statistical analysis}

Demographic data were presented as mean \pm standard deviation and 95\% confidence intervals $(\mathrm{Cl})$ or median and range. Statistical significance was evaluated in univariate analyses using chi-square test, Fisher's exact test and Mann-Whitney $U$ test. A matrix was created to illustrate the impact of combinations of different physiological and biochemical factors at the time of RRT on subsequent outcome.

Multivariate logistic regression analyses were conducted to identify independent predictors of all-cause ICU and hospital mortality and to obtain the odds ratios (ORs). Variables that were found to be significant risk factors in univariate analyses were entered simultaneously in the multivariable model (enter method). The statistical package SPSS (Version 14.0, Woking, Surrey, UK) was used for all statistical analyses. A $P<$ 0.05 was considered statistically significant.

Data related to ICU mortality are presented within this paper whereas results related to hospital mortality are available as supplementary tables in Additional data file 1 with the online version of this paper.

\section{Results \\ Patient characteristics}

For AKI, 1847 patients received RRT of whom 1473 patients (79.8\%) were treated with a continuous mode alone (continuous arterio-venous haemofiltration or continuous veno-venous haemo(dia)filtration), 95 patients $(5.1 \%)$ received intermittent haemodialysis (HD) and 12 patients $(0.6 \%)$ had peritoneal dialysis (PD) alone. Two hundred and forty patients (13.0\%) were treated with a combination of a continuous mode followed by HD. One patient had incomplete data related to the type of RRT. ICU mortality was $54.1 \%$ and hospital mortality was $61.6 \%$. ICU survivors were younger and less sick on admission to the ICU as evidenced by a significantly lower APACHE II score and fewer failed organ systems (Table 1). In addition, they had less pre-existing chronic illnesses. During their stay in ICU, they needed ventilation less often and had fewer organ failures.

RRT is one of five criteria for the diagnosis of AKI stage III and 935 patients in this study fulfilled the creatinine criteria for AKI stage III but were not treated with RRT. Direct comparison confirmed that patients with AKI stage III on RRT had a significantly higher ICU and hospital mortality than patients with AKI III not treated with RRT (Table 2). However, patients on RRT were sicker as evidenced by higher APACHE II and SOFA scores on admission to the ICU, more failed organ systems whilst in ICU and a higher need for mechanical ventilation.

Only 573 of all 1847 patients who received RRT, fulfilled the creatinine criteria for $\mathrm{AKI}$ stage III. Of the remaining patients, 691 were oliguric with a urine output less than $400 \mathrm{ml} /$ day and would have (probably) fulfilled the urine criteria for AKI stage III. The remaining 583 patients had RRT without a $300 \%$ change in creatinine or a creatinine of $354 \mu \mathrm{mol} / \mathrm{L}$ or more (as per AKI stage III classification) or without being oliguric.

\section{Parameters at time of initiation of RRT}

Among patients treated with RRT, ICU survivors were characterised by a significantly higher mean serum $\mathrm{pH}$, higher mean arterial blood pressure, fewer failed organ systems and a higher serum creatinine value at time of initiation of RRT compared with non-survivors (Table 1). There was no significant difference in mean serum urea, $\mathrm{HCO}_{3}$ and $\mathrm{K}+$ levels between ICU survivors and non-survivors. Respiratory failure was the most common associated organ failure at the time of RRT (52.2\%), followed by cardiovascular failure in $37.3 \%$ of patients. Only $5.6 \%$ of patients had hepatic failure when RRT was started but ICU mortality in this group was high at $76.9 \%$.

Of all survivors, $6.4 \%$ had intermittent haemodialysis compared with only $4.1 \%$ of the non-survivors. There was no other significant difference in the types of RRT between survivors and non-survivors.

The majority of patients (72.3\%) were initiated on RRT within the first two days after admission to ICU. Of these patients, $49.2 \%$ survived to discharge from ICU. In contrast, among patients who started RRT six days or longer after admission to ICU only $36.9 \%$ survived (Table 1 ).

Using serum urea of $27.1 \mathrm{mmol} / \mathrm{L}(76 \mathrm{mg} / \mathrm{dL})$ as a cut-off level between early and late RRT (as suggested by Liu and colleagues [9]), we found no significant difference in ICU and hospital outcome between both groups (Table 3 and Table S1 in Additional data file 1). Similarly, a serum urea cut-off of 24.2 $\mathrm{mmol} / \mathrm{L}$ (as per Bagshaw and colleagues [11]) did not differentiate between survivors and non-survivors either.

Univariate analysis showed that at the time of initiation of RRT, the following factors were associated with an increased risk of dying: low serum $\mathrm{pH}$, oligoanuria, low serum $\left(\mathrm{HCO}_{3}\right)$, total SOFA score above 12, mean arterial blood pressure of 65 $\mathrm{mmHg}$ or less, cardiovascular SOFA score above 2, respiratory SOFA score above 2, neurological SOFA score above 2, different types of organ failures and a lower serum creatinine level (Table 3 and Table S1 in Additional data file 1). The presence of more than one risk factor increased the risk of death further. Different combinations of risk factors were associated with different mortality rates (Table 4 and Table S2 in Additional data file 1). 
Critical Care Vol 13 No 6 Ostermann and Chang

Table 1

\begin{tabular}{|c|c|c|c|c|}
\hline Parameter & $\begin{array}{l}\text { ICU } \\
\text { Survivors } \\
(\mathrm{n}=848)\end{array}$ & $\begin{array}{l}\text { ICU } \\
\text { Non-survivors } \\
(n=999)\end{array}$ & $P$ & OR $(95 \% \mathrm{Cl})$ \\
\hline \multicolumn{5}{|c|}{ Parameters on admission to ICU } \\
\hline Age, Mean (SD) & $57.96(17.01)$ & $61.89(14.19)$ & $<0.0001$ & \\
\hline Male sex & $558(65.9 \%)$ & $684(68.4 \%)$ & 0.23 & \\
\hline \multicolumn{5}{|l|}{ Reason for ICU admission } \\
\hline Sepsis & $521(28.2 \%)$ & $316(60.7 \%)$ & & \\
\hline Cardiac surgery & $115(13.6 \%)$ & $121(12.1 \%)$ & & \\
\hline COPD/asthma & $203(11 \%)$ & $117(57.6 \%)$ & & \\
\hline Cardiac disease (non-surgery) & $233(12.6 \%)$ & $137(58.8 \%)$ & & \\
\hline Post-abdominal surgery & $109(5.9 \%)$ & $77(70.6 \%)$ & & \\
\hline Neurological (non-surgical) & $64(3.5 \%)$ & $13(20.3 \%)$ & & \\
\hline Trauma & $28(1.5 \%)$ & $16(57.1 \%)$ & & \\
\hline APACHE II score & $21(1-43)$ & $24(3-52)$ & 0.0001 & \\
\hline \multicolumn{5}{|l|}{ Median (range) } \\
\hline SOFA score & $10(2-20)$ & $11(0-22)$ & 0.3 & \\
\hline \multicolumn{5}{|l|}{ Median (range) } \\
\hline Number of failed organs* & $1(0-5)$ & $2(0-6)$ & $<0.0001$ & \\
\hline \multicolumn{5}{|l|}{ Median (range) } \\
\hline Emergency surgery & $93(11 \%)$ & $150(15.0 \%)$ & 0.01 & \\
\hline $\mathrm{Hb} \leq 9 \mathrm{~g} / \mathrm{dL}$ & $243(28.7 \%)$ & $253(25.3 \%)$ & 0.11 & \\
\hline Pre-existing chronic illness & $174(20.5 \%)$ & $295(29.5 \%)$ & $<0.0001$ & \\
\hline \multicolumn{5}{|l|}{ Parameters during stay in ICU } \\
\hline Ventilated & $710(83.7 \%)$ & $977(97.8 \%)$ & $<0.0001$ & $8.63(5.45-13.68)$ \\
\hline Maximum OF in ICU & $2(0-5)$ & $3(0-6)$ & 0.02 & \\
\hline \multicolumn{5}{|l|}{ Median (range) } \\
\hline \multicolumn{5}{|l|}{ Parameters at time of RRT } \\
\hline \multicolumn{5}{|c|}{ Interval between ICU admission and RRT } \\
\hline$<3$ days & $657(77.5 \%)$ & $679(68 \%)$ & $<0.0001$ & \\
\hline 3-5 days & $97(11.4 \%)$ & $159(15.9 \%)$ & ( $<3$ days $v s ~ \geq 3$ days) & \\
\hline $6-10$ days & $73(8.6 \%)$ & $101(10.1 \%)$ & & \\
\hline$>10$ days & $21(2.5 \%)$ & $60(6.0 \%)$ & & \\
\hline
\end{tabular}

Days between biochemical diagnosis of AKI III and RRT

Same day or before

1-2 days later

3-5 days later

6-10 days later

$\begin{array}{ll}1,568(84.9 \%) & 832(53.1 \%) \\ 67(3.6 \%) & 44(65.7 \%) \\ 37(2.0 \%) & 24(64.9 \%) \\ 69(3.7 \%) & 32(46.4 \%)\end{array}$




\section{Characteristics of patients on RRT}

$>10$ days later

Biochemistry, Mean (SD)

Serum urea (mmol/L) (excl $31 \mathrm{pts})$

Serum creatinine (umol/L) (excl $50 \mathrm{pts}$ )

$\mathrm{pH}$ (excl 29 pts)

Serum $\mathrm{HCO}_{3}$ (umol/L) (excl 572 pts)

Serum K (mmol/L) (excl 30 pts)

\section{Organ failure}

CVS failure

RS failure

Gl failure

NEURO failure

HAEM failure

HEP failure

CVS + RS failure

Total OF (excluding renal failure)

Median (range)

MAP (mm Hg) (excl 29 pts)

Oligoanuria (urine $\leq 400 \mathrm{ml} / \mathrm{hr}$ )

Median SOFA score [range]

\section{Type of RRT}

CRRT alone

IHD alone

PD alone

CRRT + IHD

CRRT + PD

$106(5.7 \%)$

$57(53.8 \%)$

$\begin{array}{lcl}24.1(13.6) & 24.23(13.21) & 0.84 \\ 370.9(269.9) & 312(177.9) & <0.0001 \\ 7.34(0.13) & 7.27(0.16) & <0.0001 \\ 22.1(5.94) & 20.05(6.24) & 0.11 \\ 4.55(1.11) & 4.63(1.06) & 0.17\end{array}$

$\begin{array}{lcll}243(28.7 \%) & 446(44.6 \%) & <0.0001 & 2.01(1.65-2.44) \\ 349(41.2 \%) & 615(61.6 \%) & <0.0001 & 2.29(1.90-2.76) \\ 129(15.2 \%) & 299(29.9 \%) & <0.0001 & 2.38(1.89-3.00) \\ 50(5.9 \%) & 160(16.0 \%) & <0.0001 & 3.04(2.18-4.24) \\ 62(7.2 \%) & 116(11.6 \%) & 0.002 & 1.67(1.21-2.30) \\ 24(2.8 \%) & 80(8.0 \%) & <0.0001 & 2.99(1.88-4.76) \\ 92(10.9 \%) & 260(26.0 \%) & <0.0001 & 2.89(2.23-3.74)\end{array}$

$2(0-5)$

$76.6(26.1)$

$402(47.3 \%)$

$10[0-21]$

$\begin{array}{cc}2(0-6) & <0.0001 \\ 60.5(21.01) & <0.0001 \\ 597(59.8 \%) & <0.0001 \\ 12[0-21] & <0.0001\end{array}$

$1.65(1.37-1.98)$

* excluding renal failure. $\mathrm{AKI}=$ acute kidney injury; $\mathrm{APACHE}=$ acute physiology and chronic health evaluation score; $\mathrm{Cl}=\mathrm{confidence}$ interval; $\mathrm{COPD}=$ chronic obstructive pulmonary disease; $\mathrm{CRRT}=$ continuous renal replacement therapy; $\mathrm{CVS}=$ cardiovascular; excl = excluding; $\mathrm{Gl}=$ gastrointestinal; $\mathrm{HAEM}=$ haematological; $\mathrm{Hb}=$ haemoglobin; HEP = liver; ICU = intensive care unit; $\mathrm{IHD}=$ intermittent haemodialysis; MAP = mean arterial blood pressure; $\mathrm{NEURO}=$ neurological; $\mathrm{OF}=$ organ failure; $\mathrm{OR}=$ odds ratio; $\mathrm{PD}=$ peritoneal dialysis; $\mathrm{pts}=\mathrm{patients;} \mathrm{RRT} \mathrm{=} \mathrm{renal}$ replacement therapy; RS = respiratory; SD = standard deviation; SOFA score $=$ Sequential Organ Failure Assessment score.

\section{Timing of RRT in relation to onset of AKI III}

Patients who received RRT before they met the creatinine criteria for AKI stage III (serum creatinine $\geq 354 \mu \mathrm{mol} / \mathrm{L}(\geq 4.0$ $\mathrm{mg} / \mathrm{dL}$ ) or a rise in serum creatinine by more than $300 \%$ from baseline) had a significantly lower ICU mortality than patients who were started on RRT on the day when they met the AKI stage III criteria (49.8\% versus 64.6\%; $P<0.0001$ ). This group also had a better ICU outcome compared with patients in whom RRT was initiated after the AKI criteria were fulfilled (49.8\% versus $56.3 \%)$ but this difference did not reach statistical significance $(P=0.05)$.

\section{Multivariate analysis}

In a multivariate analysis, mechanical ventilation and associated neurological failure on the day of RRT were the strongest independent risk factors for ICU and hospital mortality, fol- 
Table 2

\begin{tabular}{|c|c|c|c|}
\hline Factor & $\begin{array}{c}\text { AKI III } \\
\text { on RRT } \\
(n=1847)\end{array}$ & $\begin{array}{c}\text { AKI III } \\
\text { without RRT } \\
(n=935)\end{array}$ & $P$ \\
\hline Male sex & $1242(67.2 \%)$ & $660(70.6 \%)$ & 0.08 \\
\hline Age & $60.09(15.68)$ & $63.14(15.42)$ & 0.28 \\
\hline \multicolumn{4}{|l|}{ Mean (SD) } \\
\hline APACHE II on admission to ICU & $22(1-52)$ & $19(2-46)$ & $<0.0001$ \\
\hline Median (range) & $22.92(7.80)$ & $19.84(6.63)$ & \\
\hline \multicolumn{4}{|l|}{ Mean (SD) } \\
\hline SOFA score on admission to ICU & $10(0-22)$ & $7(0-17)$ & 0.0063 \\
\hline Median (range) & $10.23(3.18)$ & $7.61(2.96)$ & \\
\hline \multicolumn{4}{|l|}{ Mean (SD) } \\
\hline Organ failure on admission to ICU* & $2(0-6)$ & $1(0-4)$ & $<0.0001$ \\
\hline Median (range) & $1.72(1.20)$ & $1.11(0.95)$ & \\
\hline \multicolumn{4}{|l|}{ Mean (SD) } \\
\hline Maximum organ failure during ICU* & $2(0-6)$ & $2(0-6)$ & \\
\hline Median (range) & $2.27(1.17)$ & $1.67(0.97)$ & $<0.0001$ \\
\hline \multicolumn{4}{|l|}{ Mean (SD) } \\
\hline Mechanical ventilation & $1,687(91.3 \%)$ & $729(77.97 \%)$ & $<0.0001$ \\
\hline Pre-existing chronic illness & $469(25.4 \%)$ & $238(25.5 \%)$ & 0.97 \\
\hline $\mathrm{Hb} \leq \mathbf{9} \mathrm{g} / \mathrm{dl}$ on admission to ICU & $496(26.9 \%)$ & $193(20.6 \%)$ & 0.0004 \\
\hline Cardiac surgery & $236(12.8 \%)$ & 95 (10.2\%) & 0.051 \\
\hline \multicolumn{4}{|l|}{ Mortality } \\
\hline ICU mortality & 999 (54.1\%) & 380 (40.6\%) & $<0.0001$ \\
\hline Hospital mortality & $1138(61.6 \%)$ & $472(50.5 \%)$ & $<0.0001$ \\
\hline
\end{tabular}

* excluding acute kidney injury. AKI = acute kidney injury; APACHE = acute physiology and chronic health evaluation score; Hb = haemoglobin; $\mathrm{ICU}=$ intensive care unit; RRT = renal replacement therapy; SD = standard deviation; SOFA score = Sequential Organ Failure Assessment score.

lowed by liver and gastrointestinal failure, haematological failure and pre-existing chronic health problems (Table 5 and Table S3 in Additional data file 1). A higher serum $\mathrm{pH}$ was independently associated with a better outcome. Serum creatinine and urea concentrations on the day of RRT exhibited opposite associations: a raised urea and a low creatinine concentration were independent risk factors for dying. Gender, non-surgical admission, emergency surgery and cardiac surgery were not independently associated with ICU and hospital mortality.

\section{Progress after initiation of RRT}

Due to the dynamic nature of critical illness, prognosis of patients on RRT not only depends on severity of illness at time of initiation of RRT but also on subsequent progress. Patients who developed more organ failure in the 48-hour period after starting RRT had a significantly higher ICU mortality than patients without any deterioration, ie. patients who still had the same number of or fewer failed organ systems (Table 6). The same trend was seen when comparing the serum $\mathrm{pH}$ at initiation of RRT with the $\mathrm{pH}$ on the following day. Patients whose $\mathrm{pH}$ fell had a significantly higher ICU mortality than those in whom serum $\mathrm{pH}$ remained unchanged or rose.

One hundred and thirty-nine RRT patients survived their stay in the ICU but died later in hospital. The exact causes of death are not available. However, 42 patients (30.2\%) had pre-existing chronic illnesses. Furthermore, at time of discharge from the ICU, 65 patients (46.7\%) had persistent severe AKI as evidenced by a serum urea of $36 \mathrm{mmol} / \mathrm{L}$ or more, serum creatinine of $310 \mu \mathrm{mol} / \mathrm{L}$ or more or ongoing need for RRT. 
Table 3

\begin{tabular}{|c|c|c|c|c|}
\hline $\begin{array}{l}\text { Variables } \\
\text { on day of RRT }\end{array}$ & $\begin{array}{l}\text { Incidence } \\
(n=1847)\end{array}$ & $\begin{array}{l}\text { ICU } \\
\text { mortality }\end{array}$ & $P$ & $\begin{array}{l}\text { OR } \\
(95 \% \mathrm{Cl})\end{array}$ \\
\hline \multicolumn{5}{|c|}{ Serum creatinine $(\mu \mathrm{mol} / \mathrm{L})$} \\
\hline$\leq 200$ & $456(24.7 \%)$ & $255(55.9 \%)$ & $(\leq 500$ vs $>500)$ & \\
\hline$>200-500$ & $1064(57.6 \%)$ & $609(57.2 \%)$ & $<0.0001$ & \\
\hline$>500$ & $278(15.1 \%)$ & $102(36.7 \%)$ & & $2.27(1.75-2.96)$ \\
\hline$\leq 309$ & $953(51.6 \%)$ & $564(59.2 \%)$ & $<0.0001$ & \\
\hline >309 (50 data missing) & $848(45.9 \%)$ & $404(47.6 \%)$ & & $1.59(1.32-1.92)$ \\
\hline \multicolumn{5}{|l|}{ Serum pH } \\
\hline$<7.2$ & $397(21.5 \%)$ & $294(74.1 \%)$ & (<7.2 vs & \\
\hline $7.2-7.35$ & $675(36.5 \%)$ & $385(57.0 \%)$ & $\geq 7.2)<0.0001$ & \\
\hline$>7.35$ (28 data missing) & $747(40.4 \%)$ & $299(40.0 \%)$ & & $0.32(0.25-0.42)$ \\
\hline \multicolumn{5}{|l|}{ Serum $\mathrm{K}^{+}(\mathrm{mmol} / \mathrm{L})$} \\
\hline$\leq 6$ & $1634(88.5 \%)$ & $877(53.7 \%)$ & & \\
\hline$>6$ (28 data missing) & $185(10.0 \%)$ & $101(54.6 \%)$ & 0.82 & $0.96(0.71-1.31)$ \\
\hline \multicolumn{5}{|l|}{ Urine output } \\
\hline$<400 \mathrm{ml} / 24$ hours & $994(53.8 \%)$ & $595(59.9 \%)$ & & \\
\hline$\geq 400 \mathrm{ml} / 24$ hours & $853(46.2 \%)$ & $404(47.4 \%)$ & $<0.0001$ & $1.66(1.38-1.99)$ \\
\hline \multicolumn{5}{|l|}{ Serum $\mathrm{HCO}_{3}(\mathrm{mmol} / \mathrm{L})$} \\
\hline$<10$ & $50(2.7 \%)$ & $37(74 \%)$ & (<15 vs & \\
\hline $10-14.9$ & $146(7.9 \%)$ & $103(70.5 \%)$ & $\geq 15)$ & \\
\hline$\geq 15$ (577 data missing) & $1074(58.1 \%)$ & $565(52.6 \%)$ & $<0.0001$ & $2.25(1.62-3.14)$ \\
\hline \multicolumn{5}{|l|}{ Serum urea $(\mathrm{mg} / \mathrm{dL})$} \\
\hline$\leq 27.1 \mathrm{mmol} / \mathrm{L}$ & $1186(64.2 \%)$ & $641(54.0 \%)$ & 1 & \\
\hline$>27.1 \mathrm{mmol} / \mathrm{L}$ & $661(35.8 \%)$ & $358(54.2 \%)$ & & \\
\hline \multicolumn{5}{|l|}{ (30 data missing) } \\
\hline \multicolumn{5}{|l|}{ MAP (mmHg) } \\
\hline$\leq 65$ & $1,116(60.4 \%)$ & $718(64.3 \%)$ & & \\
\hline$>65$ (28 data missing) & 703 (38.1\%) & $260(37.0 \%)$ & $<0.0001$ & $3.07(2.53-3.74)$ \\
\hline \multicolumn{5}{|l|}{ SOFA CVS score } \\
\hline 0 & $270(14.6 \%)$ & $73(27 \%)$ & SOFA $\leq 2$ & \\
\hline 1 & $206(11.2 \%)$ & $77(37.4 \%)$ & versus & \\
\hline 2 & $494(26.7 \%)$ & $253(51.2 \%)$ & SOFA $>2$ & \\
\hline 3 & 0 & - & & \\
\hline 4 & $877(47.5 \%)$ & $596(67.96 \%)$ & $<0.0001$ & $2.98(2.47-3.61)$ \\
\hline \multicolumn{5}{|l|}{ SOFA RESP score } \\
\hline 0 & $21(1.1 \%)$ & 17 (80.95\%) & & \\
\hline 1 & 0 & - & & \\
\hline 2 & $225(12.2 \%)$ & $43(19.1 \%)$ & & \\
\hline 3 & 0 & - & & \\
\hline 4 & $1601(86 \%)$ & $939(58.7 \%)$ & $<0.0001$ & $4.4(3.23-5.98)$ \\
\hline \multicolumn{5}{|l|}{ SOFA NEURO score } \\
\hline 0 & $1352(73.2 \%)$ & $686(50.7 \%)$ & & \\
\hline
\end{tabular}


Table 3 (Continued)

\section{Parameters at initiation of RRT and ICU outcomeay}

2

3

4

SOFA COAG score

\section{0}

1

2

3

4

SOFA LIVER score

\section{0}

1

2

3

4

Total SOFA score

$\leq 12$

$>12$ (21 data missing)

Organ failure

CVS failure

No CVS failure

RS failure

No RS failure

Gl failure

No Gl failure

NEURO failure

No NEURO failure

HAEM failure

No HAEM failure

HEP failure

No HEP failure

CVS + RS failure

No CVS + no RS failure

Total number of failed organs

1

2

3

$>3$
$109(5.9 \%)$

$145(7.9 \%)$

$173(9.4 \%)$

$739(40 \%)$

597 (32.3\%)

$511(27.7 \%)$

0

$1125(60.9 \%)$

$206(11.2 \%)$

$140(7.6 \%)$

$188(64.4 \%)$

$84(4.5 \%)$

$1149(62.2 \%)$

$677(36.7 \%)$

$689(37.3 \%)$

$1,158(62.7 \%)$

964 (52.2\%)

$883(47.8 \%)$

$428(23.2 \%)$

$1419(76.8 \%)$

$210(11.4 \%)$

1,637 (88.6\%)

$180(9.7 \%)$

$1,667(90.3 \%)$

104 (5.6\%)

1743 (94.4\%)

$352(19.1 \%)$

546 (29.6\%)

$129(6.98 \%)$

437 (23.7\%)

683 (37.0\%)

$389(21.1 \%)$

$209(11.3 \%)$
30 (44.1\%)

59 (54.1\%)

$88(60.7 \%)$

$136(78.6 \%)$

368 (49.8\%)

331 (55.4\%)

300 (58.7\%)

N/A

550 (48.9\%)

$129(62.6 \%)$

292 (15.8\%)

84 (60.0\%)

$48(57.1 \%)$

0.133

$1.25(0.94-1.66)$

517 (45.0\%)

$465(68.7 \%)$

$<0.0001$

$0.37(0.31-0.46)$

$446(64.7 \%)$

$553(47.8 \%)$

$<0.0001$

$2.01(1.65-2.44)$

615 (63.8\%)

384 (43.5\%)

$<0.0001$

$2.29(1.90-2.76)$

299 (69.9\%)

$<0.0001$

2.38 (1.89-3.00)

700 (49.3\%)

$160(76.2 \%)$

$<0.0001$

$3.04(2.18-4.24)$

839 (51.3\%)

$118(65.6 \%)$

$881(52.8 \%)$

0.0012

$1.70(1.23-2.34)$

80 (76.9\%)

$919(52.7 \%)$

$<0.0001$

$2.99(1.88-4.76)$

$260(73.9 \%)$

198 (36.3\%)

$<0.0001$

4.97 (3.70-6.67)

$\mathrm{Cl}=$ confidence interval; COAG = coagulation; CVS = cardiovascular; excl = excluding; GI = gastrointestinal; $\mathrm{HAEM}=$ haematological; $\mathrm{HEP}=$ liver; ICU = intensive care unit; $M A P=$ mean arterial blood pressure; NEURO = neurological; OR = odds ratio; pts = patients; $R R T=$ renal replacement therapy; RESP/RS = respiratory; SOFA score = Sequential Organ Failure Assessment score; vs = versus. 


\begin{tabular}{|c|c|c|c|c|c|c|c|}
\hline Parameter at time of RRT & Serum $\mathrm{pH}<7.2$ & $\begin{array}{c}\text { Urine } \\
<400 \mathrm{ml} / 24 \text { hours }\end{array}$ & SOFA score $>12$ & $\begin{array}{l}\text { MAP } \\
\leq 65\end{array}$ & CVS failure & RESP failure & HEP failure \\
\hline Serum $\mathrm{pH}<7.2$ & $74.10 \%$ & & & & & & \\
\hline Urine $<400 \mathrm{ml} / 24$ hours & $79.10 \%$ & $59.90 \%$ & & & & & \\
\hline SOFA score $>12$ & $84.50 \%$ & $72.80 \%$ & $68.70 \%$ & & & & \\
\hline MAP $\leq 65$ & $77.30 \%$ & $69.50 \%$ & $76.50 \%$ & $64.60 \%$ & & & \\
\hline CVS failure & $75.20 \%$ & $70.40 \%$ & $76 \%$ & $71 \%$ & $64.70 \%$ & & \\
\hline RESP failure & $79 \%$ & $70.50 \%$ & $75 \%$ & $72.30 \%$ & $73.90 \%$ & $63.80 \%$ & \\
\hline HEP failure & $92 \%$ & $77 \%$ & $80.90 \%$ & $85.30 \%$ & $88.70 \%$ & $83.90 \%$ & $76.90 \%$ \\
\hline Total OF 1 * & $61.10 \%$ & $37.40 \%$ & $52 \%$ & $50.70 \%$ & - & - & - \\
\hline Total OF 2 * & $69.90 \%$ & $56.60 \%$ & $60 \%$ & $60.30 \%$ & & & \\
\hline Total OF 3 * & $74.40 \%$ & $69.50 \%$ & $73.30 \%$ & $72 \%$ & & & \\
\hline Total OF $>3$ * & $89.70 \%$ & $89.10 \%$ & $89.20 \%$ & $88.80 \%$ & & & \\
\hline \multicolumn{8}{|l|}{ Days since ICU admission } \\
\hline$<3$ & $71.30 \%$ & $57.10 \%$ & $67.70 \%$ & $61.30 \%$ & $63.40 \%$ & $62.60 \%$ & $75.60 \%$ \\
\hline 5-Mar & $94.70 \%$ & $69.70 \%$ & $71.80 \%$ & $74 \%$ & $71.40 \%$ & $65.20 \%$ & $76.90 \%$ \\
\hline 10-Jun & $83.30 \%$ & $64.80 \%$ & $65.70 \%$ & $69.10 \%$ & $65.90 \%$ & $59.70 \%$ & $75 \%$ \\
\hline$>10$ & $84.60 \%$ & $81.80 \%$ & $78.60 \%$ & $85.40 \%$ & $76.90 \%$ & $76.70 \%$ & $100 \%$ \\
\hline
\end{tabular}

* excluding acute kidney injury. CVS = cardiovascular; HEP = liver; ICU = intensive care unit; MAP = mean arterial blood pressure in mmHg; OF $=$ failed organ system(s); RESP = respiratory; RRT = renal replacement therapy; SOFA = Sequential Organ Failure Assessment.

\section{Discussion}

This study confirms that timing of RRT in critically ill patients with $\mathrm{AKI}$ remains a very complex process. Risk factors for death in the ICU were a low serum $\mathrm{pH}$, oligoanuria, mechanical ventilation and associated organ failure at time when RRT was initiated. Failure to correct acidosis or development of new organ failure within 48 hours after RRT was started, were additional markers of a poor outcome.

Despite the fact that RRT has become an integral part of modern critical care, identifying the optimal timing and optimal indication for RRT remains a difficult problem in clinical practice [17]. The literature contains several studies, which show a survival benefit with early initiation of RRT $[9,11]$. However, as documented by Bagshaw and colleagues, the differentiation between 'early' and 'late' RRT is variable and usually based on arbitrary thresholds in traditional parameters such as serum urea, serum creatinine, urine output, time from ICU admission or time from diagnosis of AKI [11]. Most evidence in favour of 'early' RRT stems from retrospective observational studies. These studies are open to the criticism that improved outcomes with 'early' RRT may simply reflect inclusion of patients with a lesser degree of organ injury and better prognosis regardless of treatment strategies. Secondly, the validity of retrospective observational data may be limited by the exclusion of patients with AKI who met criteria for early initiation of RRT but never received it. Finally, in clinical practice, it is likely that 'earlier' initiation of RRT is prompted by volume overload and/ or severe electrolyte and metabolic disturbances, whereas 'late' initiation of renal support is triggered by progressive uraemia. Whether there is a relation between these different indications and outcome is not known. The literature contains four randomized controlled trials in medical patients, post-cardiac surgery and in patients with severe sepsis [18-21] of which one is only available in abstract form [21]. Although three studies showed a survival benefit with early initiation, the exact criteria for 'early' differed in all studies. Thus, current data in the literature remain inadequate to answer the question related to optimal timing of RRT. If early initiation of RRT is indeed advantageous, it remains unclear whether this benefit may be attributable to earlier clearance of uraemic toxins, earlier metabolic/uraemic control, prevention and management of volume overload, attenuation of the inflammatory process or any other effects not yet elucidated. The counterargument to a strategy of early initiation of RRT is that patients who would recover renal function with conservative treatment alone may be subjected to unnecessary risks.

In the absence of clear data whether early RRT is better than late RRT, it may be more useful to focus on identifying the optimal triggers when to start and to provide guidance towards a more individualised approach to patients with severe AKI. In 
Table 5

\begin{tabular}{|c|c|c|c|c|c|}
\hline Parameter & B & SE & $P$ & OR & $95 \% \mathrm{Cl}$ \\
\hline PH on day of RRT & -3.719 & 0.448 & $<0.0001$ & .024 & $0.01-0.06$ \\
\hline (creatinine) on day of RRT & -0.002 & 0.000 & $<0.0001$ & .998 & $0.997-0.998$ \\
\hline (urea) on day of RRT & 0.004 & 0.001 & $<0.0001$ & 1.004 & $1.002-1.005$ \\
\hline Age & 0.026 & 0.004 & $<0.0001$ & 1.026 & $1.02-1.03$ \\
\hline CVS failure on day of RRT & 0.261 & 0.127 & 0.039 & 1.299 & $1.01-1.66$ \\
\hline Oligoanuria on day of RRT & 0.469 & 0.115 & $<0.0001$ & 1.599 & $1.28-2.00$ \\
\hline RESP failure on day of RRT & 0.485 & 0.114 & $<0.0001$ & 1.624 & $1.299-2.03$ \\
\hline HAEM failure on day of RRT & 0.553 & 0.207 & 0.008 & 1.738 & $1.16-2.61$ \\
\hline Pre-existing chronic illness & 0.554 & 0.129 & $<0.0001$ & 1.740 & $1.35-2.24$ \\
\hline GI failure on day of RRT & 0.714 & 0.135 & $<0.0001$ & 2.042 & $1.57-2.66$ \\
\hline HEP failure on day of RRT & 0.893 & 0.273 & 0.001 & 2.441 & $1.43-4.17$ \\
\hline NEURO failure on day of RRT & 0.908 & 0.197 & $<0.0001$ & 2.479 & $1.69-3.64$ \\
\hline Ventilated & 1.797 & 0.270 & $<0.0001$ & 6.029 & $3.56-10.23$ \\
\hline male gender & 0.153 & 0.120 & 0.200 & 1.166 & $0.92-1.47$ \\
\hline post non-surgical admission & 0.217 & 0.191 & 0.255 & 1.243 & $0.86-1.81$ \\
\hline post cardiac surgery & -0.062 & 0.211 & 0.770 & .940 & $0.62-1.42$ \\
\hline post-emergency surgery & 0.399 & 0.212 & 0.060 & 1.491 & $0.98-2.26$ \\
\hline Constant & 26.653 & 3.279 & $<0.0001$ & $\begin{array}{l}37587255 \\
4286.662\end{array}$ & \\
\hline
\end{tabular}

Exclusion of patients with missing data for creatinine, urea, MAP, $\mathrm{pH}$; patients included $\mathrm{n}=1794$. The area under the receiver operator characteristic curve was 0.784 (Hosmer-Lemeshow chi-squares $=7.769$; two degrees of freedom, $P=0.456$ ).

$\mathrm{AKI}=$ acute kidney injury; $\mathrm{Cl}=$ confidence interval; $\mathrm{CVS}=$ cardiovascular; $\mathrm{GI}=$ gastrointestinal; HAEM = haematological; HEP = hepatic; ICU = intensive care unit; NEURO = neurological; OR = odds ratio; RESP = respiratory; RRT = renal replacement therapy; $\mathrm{SE}=\mathrm{standard}$ error.

our patient population, ICU survival was significantly better in patients who commenced RRT before they fulfilled the creatinine criteria for AKI stage III compared with those who started RRT on the same day as the criteria were fulfilled. We also showed that marked acidosis and oligoanuria at time of RRT were associated with an increased risk of dying. In contrast, patients who were initiated on RRT with a serum creatinine less than $500 \mu \mathrm{mol} / \mathrm{L}$ had a higher ICU mortality than those with a higher value. Previous studies have demonstrated similar results where both low creatinine and high urea values at

Table 6

Impact of changes after initiation of RRT on ICU outcome

\begin{tabular}{|c|c|c|c|c|}
\hline Response after initiation of RRT & Incidence & ICU mortality & $\begin{array}{c}P \\
\text { (worsened versus unchanged) }\end{array}$ & $\begin{array}{c}P \\
\text { (worsened versus improved) }\end{array}$ \\
\hline \multicolumn{5}{|c|}{ Number of failed organs 48 hours later: } \\
\hline unchanged & $583(31.6 \%)$ & $304(52.1 \%)$ & & \\
\hline increased by 1 & $272(14.7 \%)$ & $191(70.2 \%)$ & $<0.0001$ & $<0.0001$ \\
\hline increased by $\geq 2$ & $319(17.3 \%)$ & $301(94.4 \%)$ & & \\
\hline reduced & $673(36.4 \%)$ & $203(30.2 \%)$ & & \\
\hline \multicolumn{5}{|l|}{ Serum pH 24 hours later: } \\
\hline unchanged & $172(9.3 \%)$ & $86(50 \%)$ & 0.061 & 0.008 \\
\hline reduced & $755(40.9 \%)$ & $439(58.1 \%)$ & & \\
\hline increased & $920(49.8 \%)$ & $474(51.5 \%)$ & & \\
\hline
\end{tabular}


the start of RRT were associated with a worse outcome in AKI patients $[11,22,23]$. Bagshaw and colleagues analysed the data of $1260 \mathrm{ICU}$ patients treated with RRT and found that the median serum creatinine at time of RRT was $309 \mu \mathrm{mol} / \mathrm{L}$ [11]. Crude hospital mortality was $71.4 \%$ in patients who had a serum creatinine of $309 \mu \mathrm{mol} / \mathrm{L}$ or less when RRT was initiated compared with $53.4 \%$ in patients with a higher value.

Although the exact reasons for this observation are not clear, there are several plausible explanations. First, a low serum creatinine concentration may be a reflection of low muscle mass and therefore a surrogate marker of overall health status. Second, serum creatinine may also be low as a result of associated volume overload, a condition which has been found to be independently associated with mortality in ICU patients. Third, patients who start RRT with a lower serum creatinine may indeed start RRT for reasons other than azotaemia (ie. severe acidosis, fluid overload). It is possible that different indications for RRT are associated with different outcomes.

The role of serum urea as a trigger for RRT is also controversial, especially as urea is dependent on many non-renal factors $[9,22]$. Liu and colleagues analysed the data of 243 patients on RRT and found a significantly higher risk of death at 60 days when serum urea level was $27.1 \mathrm{mmol} / \mathrm{L}$ or more $(\geq 76$ $\mathrm{mg} / \mathrm{dL}$ ) at time of RRT [9]. Adjusted for age, hepatic failure, sepsis, thrombocytopenia and serum creatinine, the relative risk of death associated with initiation of RRT at a higher urea level was 1.85 (95\% Cl 1.16 to 2.96$)$. In contrast, in our significantly larger patient population, we did not find any difference when using this cut-off. Instead, in a multivariate analysis, the most important independent prognostic factors among patients on RRT were mechanical ventilation, any associated organ failure, pre-existing chronic health problems, oligoanuria and a low serum $\mathrm{pH}$ at time of RRT.

Based on our data, we conclude that the decision when to initiate RRT should depend less on serum creatinine or urea levels but more on degree of acidosis, urine output and associated organ failure. The most likely answer to the search for the optimal timing of RRT is that sick AKI patients with significant metabolic disturbances and associated organ dysfunction benefit from 'earlier' initiation, whereas RRT can be delayed in less sick patients without causing harm. The key will be to identify the optimal triggers for RRT and optimal indications for RRT to enable a more individualised approach towards patients with severe AKI. Several factors beyond urea and creatinine are important when deciding whether renal support would be beneficial which calls for a more individualised approach.

Our data also confirm that outcome not only depends on the circumstances at time of RRT but also on events occurring in the subsequent days. Patients whose serum $\mathrm{pH}$ decreased or who developed more organ failure within 24 to 48 hours after starting RRT, had a significantly higher ICU mortality than patients whose $\mathrm{pH}$ improved or who had fewer failed organ systems. Maccariello and colleagues previously described the outcomes of $260 \mathrm{ICU}$ patients with $\mathrm{AKI}$ in response to changes in associated organ dysfunction during the first three days of RRT and made similar conclusions [24]. Organ dysfunction was determined by the SOFA score (excluding renal points) on the first and third day of RRT. Patients in whom SOFA scores worsened or remained unchanged, had a poorer hospital mortality than patients in whom SOFA scores improved ( $80 \%$ versus $84 \%$ versus $61 \% ; P=0.003$ ). These results clearly illustrate that the outcome of critically ill patients on RRT does not depend on single parameters but more on combinations of various factors and physiological responses during the dynamic course of the critical illness.

There are several limitations to our study. First, as a retrospective observational study it is potentially prone to bias. Second, our database does not include any data on the exact indications for RRT, including pulmonary oedema or fluid overload. We also did not have any data on the reasons why RRT was not offered to 935 patients with AKI stage III. Third, we did not record the dose of RRT. However, recent studies showed that the correlation between dose of RRT and outcome is less clear $[7,25,26]$. In addition, we did not record any data related to daily fluid balance and are unable to comment on the impact of fluid overload. We also did not have any data prior to ICU admission, including baseline renal function. Although we excluded patients with end-stage renal failure on long-term dialysis, we do not know how many patients had pre-existing chronic kidney disease. We accept that patients with CKD may have had a higher risk of developing acute-on-chronic renal failure and needing RRT compared with patients with normal baseline renal function. We also did not have any data on causes of death. Lastly, our database includes data from a 10-year period until 1999. It is possible that the practice of RRT has changed with advances in clinical practice. Future studies, ideally prospective randomized controlled trials will have to determine whether RRT triggered by single or multiple parameters like $\mathrm{pH}$ less than 7.2 and/or urine output less than $400 \mathrm{ml} / 24$ hours indeed leads to a better outcome. With the discovery of new AKI biomarkers, it may also be possible to identify new, yet unknown triggers for RRT.

\section{Conclusions}

Using a large database of more than 40,000 patients, we showed that $4.4 \%$ of all patients admitted to ICU needed RRT for AKI. In these patients, at time of initiation of RRT, the most important independent risk factors for ICU and hospital mortality were mechanical ventilation, associated organ failure, preexisting chronic end-stage diseases and oligoanuria. A higher serum $\mathrm{pH}$ was independently associated with a better outcome whereas serum creatinine and urea values correlated poorly with outcome. Based on these data, the decision when to start RRT should be guided more by serum $\mathrm{pH}$, urine output 
and associated comorbid factors rather than categorical creatinine and urea values.

\section{Key messages}

- The practice of RRT in critically ill patients with acute kidney injury remains variable with no agreed consensus on the optimal criteria for starting.

- In patients receiving renal support, failure of other organ systems, oligo-anuria and acidosis at time of initiation of RRT and pre-existing chronic illnesses were independent risk factors for ICU and hospital mortality.

- Failure to correct acidosis and development of more

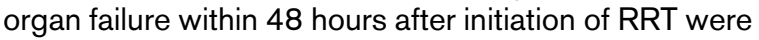
associated with increased ICU mortality.

- The decision when to start RRT for acute kidney injury should be guided more by associated dysfunction of other organ systems, urine output and serum $\mathrm{pH}$ rather than absolute serum creatinine and/or urea levels.

\section{Competing interests}

The authors declare that they have no competing interests.

\section{Authors' contributions}

$\mathrm{RC}$ organised the data collection to build the database using the Riyadh ICU Program, whose main strength is daily data collection until discharge or death, tracking the dynamic changes in the ICU. Both authors extracted the data from the database and performed the analyses. MO wrote the draft and $\mathrm{RC}$ provided critiques. Both authors approved the final manuscript.

\section{Additional files}

The following Additional files are available online:

\section{Additional file 1}

Word file containing a table that lists the parameters at initiation of renal replacement therapy (RRT) and hospital outcome (Table S1 in Additional data file 1), a table that lists the correlation between different combinations of parameters at time of RRT and hospital mortality (Table S2 in Additional data file 1), and an additional table showing the results of a multivariate analysis of parameters on the day of RRT and the associated independent risk of hospital mortality (Table S3 in Additional data file 1).

See http://www.biomedcentral.com/content/ supplementary/cc8154-S1.DOC

\section{Acknowledgements}

We wish to thank the Riyadh ICU Program Users Group for access to the data used in this study.

\section{References}

1. Mehta RL, Pascual MT, Soroko S, Savage BR, Himmelfarb J, Ikizler TA, Paganini EP, Chertow GM, for the Program of Improve Care in Acute Renal Disease: Spectrum of acute renal failure in the intensive care unit: the PICARD experience. Kidney Int 2004, 66:1613-1621.

2. Uchino S, Kellum JA, Bellomo R, Doig GS, Morimatsu H, Morgera S, Schetz M, Tan I, Bouman C, Macedo E, Gibney N, Tolwani A, Ronco C, Beginning and Ending Supportive Therapy for the Kidney (BEST Kidney) Investigators: Acute renal failure in critically ill patients: a multinational, multicenter study. JAMA 2005 , 294:813-818.

3. Ostermann M, Chang RW: Correlation between the AKI classification and outcome. Crit Care 2008, 12:R144.

4. Ostermann $\mathrm{M}$, Chang RW: Acute kidney injury in the intensive care unit according to RIFLE. Crit Care Med 2007, 35:1837-1843.

5. Overberger P, Pesacreta M, Palevsky PM, for the VA/NIH Acute Renal Failure Trial Network: Management of renal replacement therapy in acute kidney injury: a survey of practioner prescribing practices. Clin J Am Soc Nephrol 2007, 2:623-630.

6. Pannu N, Klarenbach S, Wiebe N, Manns B, Tonelli M, Alberta Kidney Disease Network: Renal replacement therapy in patients with acute renal failure: a systematic review. JAMA 2008, 299:793-805.

7. Vesconi S, Cruz DN, Fumagalli R, Kindgen-Milles D, Monti G, Marinho A, Mariano F, Formica M, Marchesi M, Rene R, Livigni S, Ronco C, Dose Response Multicentre International collaborative Initiative (DO-RE-MI Study Group): Delivered dose of renal replacement therapy and mortality in critically ill patients with acute kidney injury. Crit Care 2009, 13:R57.

8. Davenport A, Bouman C, Kirpalani A, Skippen P, Tolwani A, Mehta $\mathrm{RL}$, Palevsky PM: Delivery of renal replacement therapy in acute kidney injury: What are the key issues? Clin J Am Soc Nephrol 2008, 3:869-875.

9. Liu KD, Himmelfarb J, Paganini E, Ikizler TA, Soroko SH, Mehta RL, Chertow GM: Timing of initiation of dialysis in critically ill patients with acute kidney injury. Clin J Am Soc Nephrol 2006, 1:915-919.

10. Gibney N, Hoste E, Burdmann EA, Bunchman T, Kher V, Viswanathan R, Mehta RL, Ronco C: Timing of initiation and discontinuation of renal replacement therapy in AKI: unanswered key questions. Clin J Am Soc Nephrol 2008, 3:876-880.

11. Bagshaw SM, Uchino S, Bellomo R, Morimatsu H, Morgera S, Schetz M, Tan I, Bouman C, Macedo E, Gibney N, Tolwani A, Oudemans-van Straaten HM, Ronco C, Kellum JA, Beginning and Ending Supportive Therapy for the Kidney (BEST Kidney) Investigators: Timing of renal replacement therapy and clinical outcomes in critically ill patients with severe acute kidney injury. J Crit Care 2009, 24:129-140.

12. Seabra VF, Balk EM, Liangos $O$, Sosa MA, Cendoroglo M, Jaber $\mathrm{BL}$ : Timing of renal replacement therapy initiation in acute renal failure: a meta-analysis. $A m$ J Kidney Dis 2008, 52:272-284

13. Kellum JA, Mehta RL, Levin A, Molitoris BA, Warnock DG, Shah SV, Joannidis M, Ronco C, for the Acute Kidney Injury Network (AKIN): Development of a clinical research agenda for acute kidney injury using an international interdisciplinary three-step modified Delphi process. Clin J Am Soc Nephrol 2008, 3:887-894.

14. Mehta RL, Kellum JA, Shah SV, Molitoris BA, Ronco C, Warnock DG, Levin A: Acute Kidney Injury Network (AKIN): report of an initiative to improve outcomes in acute kidney injury. Critical Care 2007, 11:R31.

15. Knaus WA, Draper EA, Wagner DP, Zimmermann JE: Prognosis in acute organ-system failure. Ann Surg 1985, 202:685-693.

16. Chang RWS, Jacobs S, Lee B: Gastrointestinal dysfunction among intensive care unit patients. Crit Care Med 1987, 15:909-914. 
17. Palevsky PM: Clinical review: Timing and dose of continuous renal replacement therapy in acute kidney injury. Crit Care 2007, 11:232.

18. Bouman CS, Oudemans-Van Straaten HM, Tijssen JG, Zandstra DF, Kesecioglu J: Effects of early high-volume continuous veno-venous hemofiltration on survival and recovery of renal function in intensive care patients with acute renal failure: a prospective, randomized trial. Crit Care Med 2002, 30:2205-2211

19. Pursnani ML, Hazra DK, Singh B, Pandey DN: Early haemodialysis in acute tubular necrosis. J Assoc Physicians India 1997, 45:850-852.

20. Durmaz I, Yagdi T, Calkavur T, Mahmudov R, Apaydin AZ, Posacioglu $\mathrm{H}$, Atay $\mathrm{Y}$, Engin $\mathrm{C}$ : Prophylactic dialysis in patients with renal dysfunction undergoing on-pump coronary artery bypass surgery. Ann Thorac Surg 2003, 75:859-864.

21. Koo JR, Yoon JW, Oh JE: Prospective evaluation of early continuous venovenous haemofiltration on the outcome in patients with severe sepsis or septic shock. J Am Soc Nephrol 2006, 17:50A. abstract

22. Mehta RL, Pascual MT, Gruta CG, Zhuang S, Chertow GM, for the PICARD Study Group: Refining predictive models in critically ill patients with acute renal failure. J Am Soc Nephrol 2002, 13:1350-1357.

23. Paganini EP, Halstenberg WK, Goormastic M: Risk modelling in acute renal failure requiring dialysis: The introduction of a new model. Clin Nephrol 1996, 46:206-211.

24. Maccariello E, Rocha E, Valente C, Nogueira L, Rocha PT, Bonomo H Jr, Serpa LF, Ismael M, Valença RV, Machado JE, Soares M: Effects of early changes in organ dysfunction on the outcomes of critically ill patients in need of renal replacement therapy. Clinics 2008, 63:343-350.

25. VA/NIH Acute Renal Failure Trial Network, Palevsky PM, Zhang JH, O'Connor TZ, Chertow GM, Crowley ST, Choudhury D, Finkel K, Kellum JA, Paganini E, Schein RM, Smith MW, Swanson KM, Thompson BT, Vijayan A, Watnick S, Star RA, Peduzzi P: Intensity of renal support in critically ill patients with acute kidney injury. N Engl J Med 2008, 359:7-20.

26. The RENAL Replacement Therapy Study Investigators: Intensity of continuous renal replacement therapy in critically ill patients. N Engl J Med 2009, 361:1627-1638. 